On the evening of the first day of the session the members present dined together at the Avenue House and a very enjoyable hour was spent.

The next meeting of the Section will take place during the spring of the current year, of which due notice will be given.

Thomas F. Holgate, Secretary of the Section.

\title{
THE PHILOSOPHY OF HYPERSPACE.
}

PRESIDENTIAL ADDRESS DELIVERED BEFORE THE AMERICAN MATHEMATICAL SOCIETY AT ITS FOURTH ANNUAL MEETING, DECEMBER 29, 1897.

BY PROFESSOR SIMON NEWCOMB.

THERE is a region of mathematical thought which might be called the fairyland of geometry. The geometer here disports himself in a way which, to the non-mathematical thinker, suggests the wild flight of an unbridled imagination rather than the sober sequence of mathematical demonstration. Imaginative he certainly does become, if we apply this term to every conception which lies outside of our human experience. Yet the results of the hypotheses introduced into this imaginary universe are traced out with all the rigor of geometric demonstration. It is quite fitting that one who finds the infinity of space in which our universe is situated too narrow for his use should, in his imaginative power, outdo the ordinary writer of fairy tales, when he evokes a universe sufficiently extended for his purposes.

The introduction of what is now very generally called hyperspace, especially space of more than three dimensions, into mathematics has proved a stumbling block to more than one able philosopher. The question whether a fourth dimension may possibly exist, and whether it can be legitimately employed for any mathematical purpose, is one on which clear ideas are not universal. I do not, however, confine the term "hyperspace" to space of more than three dimensions. A hypothesis which is simpler in its fundamental basis, and yet seems absurd enough in itself, is that of what is sometimes, improperly I think, called curved space. This also we may call hyperspace, defining the latter in general as space in which the axioms of the Euclidean geometry are not true and complete. Curved space and space of four or more dimensions are completely dis- 
tinct in their characteristics, and must therefore be treated separately.

The hypothesis of a fourth dimension can be introduced in so simple a way that it should give rise to no question or difficulty whatever. Indeed, the whole conception is so simple that I should hardly deem it necessary to explain the matter to a professional mathematical student. But as we all have to come in contact with educated men who have not had the time to completely master mathematical conceptions, and yet are interested in the fundamental philosophy of our subject, I have deemed it appropriate to present the question in what seems to me the simplest light.

The student of geometry begins his study with the theory of figures in a plane. In this field he reaches certain conclusions, among them that only one perpendicular can be drawn to a line at a given point, and that only one triangle can be erected with given sides on a given base in a given order. Having constructed this plane geometry, he passes to geometry of three dimensions. Here he enters a region. in which some of the propositions of plane geometry cease to be true. An infinity of perpendiculars can now be drawn to a given line at a given point, and an infinity of triangles can be constructed on a given base with given sides. $\mathrm{He}$ has thus considered in succession geometry of two dimensions, and then passes to geometry of three dimensions. Why should he stop there? You reply, perhaps, because there are only three dimensions in actual space. But in making hypotheses we need not limit ourselves to actualities; we can improve our methods of research, and gain clearer conceptions of the actual by passing outside and considering the possible.

For logical purposes there is no limit to the admissibility of hypotheses, provided we consider them purely as hypotheses, and do not teach that they are actual facts of the universe. It is, therefore, perfectly legitimate to inquire what our geometry would be if, instead of being confined to three dimensions, we introduced a fourth. Many curious conclusions follow. When we are confined to a plane a circle completely bounds a region within the plane, so that we cannot pass from the inside to the outside of the circle without intersecting it. Beings conscious only of two dimensions and moving only in two dimensions, and placed inside such a material circle, would find themselves completely imprisoned, with no possibility of getting outside. But give them a third dimension, with the power to move into it, and they simply step over the circle without break- 
ing it. They do not have to even touch it. Living, as we do, in space of three dimensions, the four walls, pavement and ceiling of a dungeon, confine a person so completely that there is no possibility of escaping without making an opening through the bounding surface. But give us a fourth dimension, with the faculty of moving into it, and we pass completely outside of our three dimensional universe, by a single step, and get outside the dungeons as easily as a man steps over a line drawn on the ground. Were motion in the fourth dimension possible, an object moving in that dimension by the smallest amount would be completely outside of what we recognize as the universe, and would therefore become invisible. It could then be turned around in such a way that on being brought back it would be obverted, or appear as in a looking glass. A man capable of such a motion would come back into our sight similarly obverted, his left side would now be his right, without any change having taken place in the relative positions of the particles of his body. The somerset he would have turned would have completely obverted every atom and molecule of his body without introducing any disturbance into its operations.

This possibility of obversion brings in a curious question concerning the rigor of one of the fundamental propositions in elementary geometry. Euclid proves by superposition that the two triangles in a plane having two angles and the included side equal are equal to each other. In the demonstration it is assumed that the triangles can be made congruent by simply placing one upon the other without taking it out of the plane. From this the conclusion is drawn that the same conclusion holds true if one of the triangles be obverted. But in this case they cannot be brought into congruence without taking one of them out of the plane and turning it over. The third dimension is thus assumed in geometry involving only two dimensions.

Now consider the analogous case in space. Two pyramids upon congruent bases may be proved equal by bringing them into congruence with each other. But suppose that they differ only in that one is the obverse of the other, so that they could be brought into congruence only by looking at one of them in a mirror and then placing the other into congruence with the image of the first as seen in the mirror. Would we detract from the rigor of the demonstration by assuming the possibility of such an obversion without changing the volume of the pyramid? With a fourth dimension we should have no detraction from rigor. We 
would simply obvert the pyramid as we would turn over the triangle.

The question of the fourth dimension as a reality may be considered from two points of view, its conceivability and its possible objective reality. If by conceivability we mean the power of being imaged in the mind it must be admitted that it is absolutely inconceivable. We have no difficulty in forming a visual conception of three lines passing through the same point, each of which is at right angles to the other two. Such is the familiar system of coördinate axes in space. But he who would conceive a fourth dimension must be able to imagine a fourth axis perpendicular to all three of the others. This clearly transcends all possibility even of imagination. The fourth dimension in this sense is certainly inconceivable.

The question of the objective possibility of the fourth dimension is quite a distinct one from that of its conceivability. The latter limitation upon our faculties grows out of the objective fact that we and our ancestors have had no experience of a fourth dimension; that we have always lived in a universe of three dimensions only. But we should not too readily conclude that all being is necessarily confined to these three dimensions. Those who speculate on the possible have taken great pleasure in imagining another universe alongside of our own and yet distinct from it. The mathematician has shown that there is nothing absurd or contradictory in such a supposition. But when we come to the question of physical fact we must admit that there appears to be no evidence of such a universe. If it exists, none of its agencies intrude into our own universe, at least in the opinion of sober thinkers. The intrusion of spirits from without into our world is a favorite idea among primitive men, but tends to die out with enlightenment and civilization. Yet there is nothing self-contradictory or illogical in the supposition. The fish that swims the ocean experiences objects which, to him, seem to come from outside his universe-steamships for example. If our atmosphere had been opaque to the rays of light from the sun, or even if it had been so filled with clouds and vapor that we could never see outside of it, we also should have had a similar experience. But we may be said, in a certain sense, to see through the whole of our conceivable space with the aid of our telescopes, and the general tendency of scientific thought at the present time is toward the conclusion that no natural agency of which we can trace the operation originates outside the space into which our telescopes may pene- 
trate. Our universe forms, so to speak, a closed system. This is true apparently even of agencies so subtle as those which give vibrations to ether. If there is any agency which we could imagine to connect us with an outside sphere it is certainly the luminiferous ether. But should this ether enter into a fourth dimension the intensity of light and radiant heat would diminish as the cube of the distance and not as the square. To speak more accurately, radiance emanating from an incandescent body would be entirely lost-would pass completely out of our universe. The fact that it is not lost, and indeed the general theory of the conservation of energy, shows that there is no interchange of energy between our universe and any possible one lying in another dimension of space.

We may regard the limitations of the dimensions of space to three as expressing in a certain way a physical fact. Our conception of space is originally based upon the possibility of motion. The threefold possibility of relative motion can be reduced to a physical fact in this way. Let a point be fixed at one end of a rod, the other end of which is immovably fixed to a wall. The point can then have motion over the surface of a sphere whose center is at the fixed point and whose radius is the length of the rod. Now fix one end of a second rod to another point of the plane and bring the two ends of the rods together, and fix the point on both ends; then the point can only move in a circle. Fasten it to a third point of the plane with a third rod, and it cannot move at all. But if we add a fourth dimension it could move.

The limits of space are for us simply the limits of possible motion of a material body. We can imagine a body coming from any point in three-dimensional space to us, but cannot imagine one coming from outside of such space, until we add a fourth dimension.

Our conclusion is that space of four dimensions, with its resulting possibility of an infinite number of universes alongside of our own, is a perfectly legitimate mathematical hypothesis. We cannot say whether this conception does or does not correspond to any objective reality. What we can say with confidence is that if a fourth dimension exists, our universe and every known agency in it is, by some fundamental law of its being, absolutely confined to three of the dimensions. But we must not carry a conclusion of this sort beyond the limits set by experience. When we say that experience shows that not only our material universe but all known agencies in it are, by a law of their being, 
incapable of motion in more than three dimensions we must remember that the conclusion applies only to those motions which our senses can perceive, the motions of masses in fact. There is no proof that the molecule may not vibrate in a fourth dimension. There are facts which seem to indicate at least the possibility of molecular motion or change of some sort not expressible in terms of time and three coördinates in space. If we consider those conceptions of mechanics which we derive from visible phenomena to afford a sufficient explanation of molecular action we must admit that, when the position and motion of every atom of a given substance are defined, the chemical properties of that substance are completely determined. If we take two collections of atoms of the same substance, put them together in the same way, and endow them with the same kinds of vibratory motion, we ought, on any mechanical theory of matter, to obtain substances of identical properties. Now, there seem to be reasons which I cannot stop at present to develop that might make us believe in changes of properties and attributes of substances not completely explained by molecular changes. That such is the case with vital phenomena can be demonstrated beyond doubt; that it is the case with chemical phenomena when they approach the vital character seems very probable. Certainly there is some essential difference between that form of molecular motion in which heat is commonly supposed to consist and the motion of masses. Perhaps the most remarkable of these differences consists in the relation of this motion to the ether. The motion of a mass suffers no resistance by passing through the ether with the highest astronomical velocities. Matter so rare as that of the diffuse comets may move around the sun with a speed of many miles per second without suffering the smallest resistance from the etherin a word, without any friction between the matter and the ether. But when the molecules have the motion of heat, that motion, if motion it be, is always communicated to the ether, and is radiated away from the body, which thus becomes cool. Whatever form we attribute to the energy of heat, it is certainly a form which is constantly communicated from matter to the ether by a fundamental law of matter. Consequently, if heat be really a mode of motion, as is now generally supposed by physicists, it follows that there is some essential difference between the character of this motion and the motion of the smallest masses into which matter can practically be divided. The hypothesis of vibration in the fourth dimension merely suggests the 
possibility that this kind of motion may mark what is essentially different from the motion of masses. Of course, such an hypothesis as this is not to be put forward as a theory. It must be worked out with mathematical rigor, and shown to actually explain phenomena before we assign it to any such rank.

I cannot but fear that some confusion on this subject is caused by the tendency among both geometers and psychologists to talk of space as an entity in itself. As I have already said, a fourth dimension in space is nothing more than the addition of a fourth possibility of motion to material bodies. The laws of space are only laws of relative position. Certain fundamental axioms are derived from experience, not alone individual experience, perhaps, but the experience of the race, giving rise to hereditary conceptions born in the mind and corresponding to the facts of individual experience. A tree confined to one spot, even if it had eyes to see and a brain to think could never have a conception of space. For us the limits of space are simply the limits to which we can suppose a body to move. Hence when space itself is spoken of as having possible curvatures, hills and hollows, it seems to me that this should be regarded only as a curvature, if I may use the term, of the laws of position of material bodies in space. Clifford has set forth, with great acuteness and plausibility, that the minute spaces occupied by the ultimate atoms of matter may, in this respect, have properties different from the larger space which alone makes itself known to our conceptions. If so, we should only regard this as expressive of some different law of motion, or, since motion is only change of position, of some different law of position among the molecules of bodies.

This consideration leads us to a possible form of space relations distinct from those of our Euclidean geometry, and from the hypothesis of space of more than three dimensions. I refer to what is commonly known as "curved space." The history of this conception is now so well known to mathematicians that I shall mention it only so far as is necessary to bring it to your minds. The question whether Euclid's axiom of parallels is really an independent axiom underivable from the other axioms of geometry, is one which has occupied the attention of mathematicians for centuries. Perhaps the simplest form of this axiom is that through a point in a plane one straight line and no more can be drawn which shall be parallel to a given straight line in the plane. Here we must understand that parallel lines mean 
those which never meet. The axiom, therefore, asserts that through such a point we can draw one line which shall never meet the other line in either direction, but that if we give this one line the slightest motion around the point in the plane it will meet the other in one direction or the opposite. Thus stated, the proposition seems to be an axiom, but it is an axiom that does not grow out of any other axioms of geometry. The question thus arising was attacked by Lobatchevsky in this very conclusive manner. If this axiom is independent of the other axioms of geometry then we should be able to construct a self-consistent geometrical system, in conformity to the other axioms, in which this axiom no longer held. The axiom of parallels may be deviated from in two directions. In the one it is supposed that every two lines in the plane must meet; no line parallel to another can be drawn through the same point in the plane. Deviating in the other direction we have several lines drawn through the point which never meet the given line; they diverge from it as lines on an hyperboloid may diverge.

That such possibilities transcend our ordinary notions of geometrical relations is beyond doubt, but the hypothesis of their possibility is justified by the following analogy. Let us suppose a class of beings whose movements and conceptions were wholly confined to a space of two dimensions as ours are to a space of three dimensions. Let us suppose such beings to live upon or in a plane and to have no conception of space otherwise than as plane extended space. These beings would then have a plane geometry exactly like ours. The axiom of parallels would hold for them as it does for us. But let us suppose that these beings, without actually knowing it, instead of being confined to a plane, were really confined to the surface of a sphere, a sphere such as our earth, for example. Then, when they extended their motions and observations over regions so great as a large part of the earth's surface, they would find the axiom of parallels to fail them. Two parallel lines would be only two parallel great circles, and though each were followed in a direction which would seem to be invariable they would be found to meet on opposite sides of the globe. The suggestion growing out of this consideration is : May it not be possible that we live in a space of this sort? Or, to use what seems to me to be the more accurate language : May it not be that two seemingly parallel straight lines continued indefinitely would ultimately meet or diverge? The conceptions arising in this way are certainly very interesting. If the lines would meet it can easily be shown that the total 
volume of all space is a finite quantity. The sum of the three angles of a triangle extending from star to star would then be greater than the sum of two right angles. Equally legitimate is the hypothesis that it would be less than three right angles, but in this case the total volume of space would still be infinite. Now, this is an hypothesis to be tested by experience. Unfortunately, we cannot triangulate from star to star; our limits are the two extremes of the earth's orbit. All we can say is that, within those narrow limits, the measures of stellar parallax give no indication that the sum of the angles of a triangle in stellar space differs from two right angles. If our space is elliptical, then, for every point in it-the position of our sun, for example-there would be, in every direction, an opposite or polar point whose locus is a surface at the greatest possible distance from us. A star in this point would seem to have no parallax. Measures of stellar parallax, photometric determinations and other considerations show conclusively that if there is any such surface it lies far beyond the bounds of our stellar system.

Such are the considerations by which it seems to me that speculations on this subject may legitimately be guided. The wise man is one who admits an infinity of possibilities outside the range of his experience, but who in considering actualities is not decoyed by the temptation to strain the facts of experience in order to make them accord with glittering possibilities. The experience of the race and all the refinements of modern science may be regarded as showing quite conclusively that, within the limits of our experience, there is no motion of material masses in the direction of a fourth dimension, no physical agency which we can assume to have its origin in regions to which matter cannot move, when it has three degrees of freedom. Claiming this, we must carry the claim only to the limits justified by actual experience. We have no experience of the motion of molecules ; therefore we have no right to say that those motions are necessarily confined to three dimensions. Perhaps the phenomena of radiation and electricity may yet be explained by vibration in a fourth dimension. We are justified by experience in.saying that the space relations which we gather from observation around us are valid for the greatest distances which separate us from the most distant stars. We have no right to extend the conclusion further than this. We must leave it to our posterity to determine whether, in either way, the hypothesis of hyperspace can be used as an explanation of observed phenomena. 